\title{
A enfermagem em saúde mental pós reforma psiquiátrica
}

\author{
Mental health nursing after psychiatric reform
}

La enfermería en salud mental después de la reforma psiquiátrica

Adriana dos Santos Estevam ${ }^{1 *}$, Douglas Vinícius dos Santos Feitosa ${ }^{2}$, Noemia Santos de Oliveira Silva², Sâmia Nunes de Melo $^{3}$, Ana Paula Aragão Santos ${ }^{1}$, Thaynara Fontes Almeida².

\section{RESUMO}

Objetivo: Verificar o panorama atual da enfermagem psiquiátrica/saúde mental no contexto após a reforma psiquiátrica. Métodos: Pesquisa descritiva tipo revisão integrativa e de abordagem qualitativa, baseada nas produções científicas nacionais dos últimos cinco anos disponíveis nas bases da BVS, BDENF, LILACS e index psicologia. Resultados: Foram encontrados 575 (100\%) artigos e após analise restaram 11 (1,91\%), para compor esse estudo. Observou-se que os artigos ressaltam a importância dos estudos científicos na saúde mental para a educação continuada da equipe de enfermagem e dos profissionais que têm contato com essa população. Considerações finais: Com essa mudança de panorama do cenário da saúde mental a enfermagem assumiu papel importante tanto na consolidação da Reforma Psiquiátrica quanto nas mudanças das práticas e no contexto da saúde pública e em âmbito hospitalar, enaltecendo a enfermagem como uma profissão de suma importância e tida atualmente como protagonista em todos os serviços substitutivos de atenção psicossocial.

Palavras-chave: Saúde mental, Enfermagem psiquiátrica, Avaliação de serviços de saúde.

\begin{abstract}
Objective: To verify the current state of psychiatric/mental health nursing in the context after psychiatric reform. Methods: Integrative and qualitative review type descriptive research, based on the national scientific productions of the last five years available in the bases of the VHL, BDENF, LILACS and index psychology. Results: $575(100 \%)$ articles were found and after analysis $11(1.91 \%)$ remained to compose this study. It was observed that the articles emphasize the importance of scientific studies in mental health for the continued education of nursing staff and professionals who have contact with this population. Final considerations: With this change of panorama of the mental health scenario, nursing has assumed an important role in the consolidation of the Psychiatric Reform as well as changes in practices and in the context of public health and in the hospital, praising nursing as a profession of great importance and currently regarded as a protagonist in all substitute services of psychosocial care.
\end{abstract}

Keywords: Mental health, Psychiatric nursing, Health services evaluation.

\section{RESUMEN}

Objetivo: Verificar el panorama actual de la enfermería psiquiátrica/salud mental en el contexto después de la reforma psiquiátrica. Métodos: Investigación descriptiva tipo revisión integrativa y de enfoque cualitativo, basada en las producciones científicas nacionales de los últimos cinco años disponibles en las bases de la

1 Universidade Federal de Sergipe, Aracaju-SE. *E-mail: dricaestevam@bol.com.br

2 Centro Universitário Estácio de Sergipe, Aracaju-SE.

${ }^{3}$ Faculdade Maurício de Nassau, Aracaju-SE. 
BVS, BDENF, LILACS e index psicología. Resultados: Se encontraron 575 (100\%) artículos y después de analizar quedaron $11(1,91 \%)$, para componer ese estúdio. Se observó que los artículos resaltan la importancia de los estudios científicos en la salud mental para la educación continuada del equipo de enfermería y de los profesionales que tienen contacto con esa población. Consideraciones finales: en cuanto a los cambios de las prácticas y en el contexto de la salud pública y en el ámbito hospitalario, alabando la enfermería como una profesión de suma importancia y tenida actualmente como protagonista en todos los servicios sustitutivos de atención psicosocial.

Palabras clave: Salud mental, Enfermería psiquiátrica, Investigación sobre servicios de salud.

\section{INTRODUÇÃO}

A Reforma Psiquiátrica (RP) do Brasil surgiu em um período de mobilização social em busca da democracia, sendo influenciada fortemente pelo movimento da reforma italiana. Esse modelo trouxe a negação do paradigma manicomial, mas sobretudo nos saberes e suas práticas. A partir da superação desse modelo manicomial, foram necessários outros avanços para a forma de cuidar, voltando-se a atenção para o novo modelo de atenção psicossocial, incluindo neste os serviços extra-hospitalares - o que garante a reinserção do indivíduo na comunidade (ARGILES CT, et al., 2013).

A influência italiana e os seus princípios foram incorporados no Brasil pelas portarias do Ministério da Saúde 189/1991 e 224/1992 e na criação dos novos serviços. Tendo atendimento em oficinas terapêuticas, CAPS (Centro de atenção psicossocial), hospital-dia, urgência e internação em hospital geral (BRASIL, 2004).

Durante esse tempo de luta antimanicomial, houve alguns marcos teórico-políticos que ajudaram a impulsionar à superação deste modelo, como a 8ª Conferência Nacional de Saúde em 1986, a 1ํㅡㄹ Conferência de Saúde Mental em 1987, a $2^{\underline{a}}$ Conferência Nacional de Saúde Mental em 1992, culminando na $3^{\text {a }}$ Conferência Nacional de Saúde Mental em 2001 (HIRDES AA, 2009).

Com essas mudanças no campo da assistência de Saúde Mental (SM), houve a necessidade de reorganização dos serviços e a criação de novas modalidades de atendimento terapêutico. Esse processo de transformação, bem como o movimento da luta antimanicomial implicaram em mudanças no âmbito teórico, jurídico e sociocultural, no qual passa pelo campo da construção de modelo de atenção e políticas (TAVARES CMM, et al., 2014).

Para poder readequar as necessidades em relação aos saberes e suas práticas da equipe de enfermagem, foi criado em 1980, a Escola Profissional de Enfermeiros e Enfermeiras, originado no Hospital Nacional de Alienados, onde mais tarde passou a ser chamado de Escola de Enfermagem Alfredo Pinto (MAGNAGO C; TAVARES CMM, 2012).

É no auge dessa reforma que surge esses novos modelos ou dispositivos de atenção ao indivíduo em sofrimento psíquico (LIMA DWC, et al., 2015), destacando-se alguns modelos mais presentes como o Hospital-dia, os Serviço Residencial Terapêutico (SRT), Serviços Ambulatoriais, Centros de Atenção Psicossocial (CAPS), Leito Psiquiátrico em Hospital Geral, Casa de Acolhimento Transitório, Consultório de Rua, entre outros, e maioria desses dispositivos buscam promover a reinserção social e o resgate da autonomia dos portadores de transtorno mental, o que preserva sua subjetividade e individualidade, com a aproximação e reaproximação da família e meio comunitário (AZEVEDO DM e SANTOS AT, 2012).

Esses dispositivos seguem os preceitos da lógica antimanicomial, onde busca promover a desinstitucionalização dos hospitais psiquiátricos, promovendo a mudança do modelo manicomial para 0 modelo de atenção psicossocial, juntamente, com as redes extra-hospitalares.

Esse advento compreendeu um processo político e social muito complexo, pois, envolveu a transformação de práticas, saberes, valores culturais e sociais acerca da loucura. Em 6 de abril de 2001, foi aprovado a lei 10.216, onde conferiu de fato a proteção do indivíduo em sofrimento psíquico e redimensionando o modelo antimanicomial para o modelo psicossocial (LOPES CV e REINALDO AMS, 2012). 
Nesse mesmo tempo passa a vigorar a portaria 336 de fevereiro de 2002, onde estabelece as funções, modalidades e composição das equipes atuante dentro de um CAPS. Anos depois surgiu a portaria 3.088/11, no qual traz a enfermagem como possuidora de um papel fundamental, uma vez que em todos os componentes da RAPS (rede de atenção psicossocial) preconiza a imagem e presença do enfermeiro (ESPERIDIÃO E, et al., 2013).

Esses novos modelos de SM têm como eixo a reestruturação da assistência, a criação de serviços substitutivos e fechamento dos serviços manicomiais, porém, com essas mudanças, de modelo de cuidado, surge preocupação com a formação profissional que assegure uma assistência conforme os preceitos da RP. Entretanto, na prática pode-se perceber resistência por parte de alguns profissionais, onde alguns ainda cuidam com a concepção do modelo tradicional, muitos destes por não saberem como atuar dentro desses novos modelos (SOUZA MC e AFONSO MLM, 2015).

Mediante isso, viu-se a necessidade de pesquisar na literatura o panorama atual desta nova enfermagem, observando que muitas dificuldades ainda permeiam as práticas na assistência as pessoas que tem algum tipo de doença mental. Para alcançarmos tal proposto, temos como objetivo geral verificar o panorama atual da enfermagem psiquiátrica/saúde mental no contexto atual após a reforma psiquiátrica no Brasil.

\section{MÉTODOS}

Pesquisa fundamentada em produções científicas nacionais, com abordagem qualitativa, de cunho descritivo e baseada em revisão integrativa da literatura, com o objetivo de descrever informações sobre o assunto em estudo, buscando seu conceito e esboço. A pesquisa tem como foco analisar o panorama da enfermagem em saúde mental no Brasil após a reforma psiquiátrica.

Para realizar a fundamentação do estudo seguiu-se seis etapas esquematizadas: a elaboração do tema e da questão norteadora; selecionado dados para análise dos critérios de inclusão e exclusão, levantamento da busca nas bases de dados; análise do material a serem utilizados para construção do estudo e interpretação dos resultados (MENDES KC, et al., 2008).

$\mathrm{Na}$ abordagem aos critérios de inclusão foram obtidos artigos com um intervalo máximo de 05 anos de publicação (compreendidos entre os anos de 2012 e 2017), que tragam a temática da pesquisa em estudo, publicados em português com descrição em textos completos e disponíveis online. Quanto aos critérios de exclusão segui-se a seleção criteriosa onde foram excluídos trabalhos com perfil de revisão.

Realizado levantamento dos artigos nos bancos de dados seguindo critérios que corresponderam ao objetivo da pesquisa, após análise realizada a tabulação organizada dos dados e elaborado um instrumento em forma de tabela com o objetivo e inserir os dados coletados, seguindo a ordem: autor/ano, o título do artigo e conclusão dos autores. Foi utilizado o programa Microsoft Office Word versão 2010, para análise estatística das literaturas encontradas na base de dados e realizado o cálculo tendo como base a frequência absoluta e relativa.

Para o estudo foi realizada a utilização de artigos disponíveis online, assim seguiu-se as normas que consta na lei $n^{0} 12.853 / 13$ a qual regulamenta os direitos autorais dos autores analisados. $O$ estudo considerou aos critérios de ética em pesquisa nas normas da Associação Brasileira de Normas Técnicas ABNT 10.520 e 6023, que falam sobre as regras de citações e referências, respeitando as autorias e seguindo os princípios éticos.

A realização do levantamento dos artigos executou-se utilizando os descritores ao operador boleano: "Enfermagem em saúde mental OR Enfermagem psiquiátrica AND reforma psiquiátrica, assim após análise dos artigos foram encontrados no total de 575 o qual representa $100 \%$ dos trabalhos científicos, para a busca de dados foi utilizada a Biblioteca Virtual de Saúde (BVS) utilizando os descritores: Enfermagem em saúde mental, Enfermagem psiquiátrica, Reforma psiquiátrica.

Após a leitura e utilizando os critérios de inclusão e exclusão da pesquisa foram analisados e obtido um resultado final com um total de 48 artigos. No total de 48 artigos, foram excluídos $6(1,04 \%)$ por se tratarem 
de revisão, por ter características de repetição $20(3,48 \%)$ e os que não tem relação com os objetivos da pesquisa totalizando $11(1,91 \%)$, tendo assim $11(1,91 \%)$ de artigos para serem analisados (tabela 1$)$.

Os critérios de inclusão e exclusão da pesquisa foram analisados e obtido no resultado final o total de 48 artigos. Dentre o total de 48 artigos, $6(1,04 \%)$ foram excluídos por se tratarem de revisão, $20(3,48 \%)$ por repetição, $11(1,91 \%)$ não tem relação com os objetivos totalizando $11(1,91 \%)$ artigos para a posterior análise (tabela 1).

Tabela 1 - Descrição das fontes literárias encontradas nas bases de dados após análise dos critérios de inclusão e exclusão - Brasil, 2017.

\begin{tabular}{ccc}
\hline Critérios para seleção & $\mathbf{F i}$ & $\mathbf{f i} \%$ \\
\hline Textos excluídos após aplicação dos critérios & 527 & 91,65 \\
Revisão & 6 & 1,04 \\
Repetidos & 20 & 3,48 \\
Fora dos objetivos & 11 & 1,91 \\
Total para análise $(\mathrm{n})^{*}$ & 11 & 1,91 \\
Total de literaturas ${ }^{*}$ & 575 & $100 \%$ \\
\hline
\end{tabular}

Fonte: Estevam AS, et al., 2018.

Foram analisados 11 artigos conforme alguns perfis, os quais foram organizados como: distribuição por ano de publicação entre o período de 2012 a 2017, três artigos no ano de 2012, um no ano de 2013; dois em 2014 e três no ano de 2015, mas no ano de 2016 não foram encontrados artigos similar ao perfil da pesquisa, e em 2017 foram verificadas duas publicações. Em relação aos indexos na base de dados da BVS, o perfil apresentado foi de 1 no index psicologia, 5 no LILACS e 5 na BDENF (tabela 2).

Tabela 2 - Representação da amostra da pesquisa com um total de 11 artigos, conforme base de dados BVS e ano de publicação- Brasil, 2017.

\begin{tabular}{cccc}
\hline Ano & $\begin{array}{c}\text { Quant. } \\
(\mathbf{n = 1 8})\end{array}$ & Indexo na BVS & $\begin{array}{c}\text { Quant. } \\
(\mathbf{n = 1 8})\end{array}$ \\
\hline 2012 & 3 & Index psicologia & 1 \\
2013 & 1 & LILACS & 5 \\
2014 & 2 & BDENF & 5 \\
2015 & 3 & & \\
2016 & 0 & & \\
2017 & 2 & & \\
\hline
\end{tabular}

Fonte: Estevam AS, et al., 2018.

\section{RESULTADOS}

Com os 11 artigos selecionados foi realizada a construção de um quadro, a fim de realizar uma análise dos resultados apresentados. Estes resultados foram evidenciados no quadro 1 onde consta o Autor/ano, o título do artigo e conclusão dos autores ao finalizar suas pesquisas. 


\section{Revista Eletrônica Acervo Saúde / Electronic Journal Collection Health | ISSN 2178-2091}

Quadro 1 - Distribuição dos artigos após análise dos dados evidenciados - Brasil, 2017.

\begin{tabular}{|c|c|c|}
\hline Autor (es)/ano & Titulo & Conclusão \\
\hline $\begin{array}{l}\text { AZEVEDO DM e } \\
\text { SANTOS A (2012). }\end{array}$ & $\begin{array}{l}\text { Ações de saúde mental na atenção } \\
\text { básica: conhecimento de enfermeiros } \\
\text { sobre a reforma psiquiátrica. }\end{array}$ & $\begin{array}{l}\text { Os enfermeiros alegam conhecer a RP e a importância desta na ESF. As divergências e } \\
\text { discordâncias encontradas acabam sugerindo fragilidade entre a articulação CAPS e ESF, } \\
\text { interferindo na assistência e demonstrando desconhecimento sobre o matriciamento. }\end{array}$ \\
\hline $\begin{array}{lr}\text { LOPES } & \text { CV } \\
\text { REINALDO } & \text { e } \\
(2012) . & \text { AMS }\end{array}$ & $\begin{array}{l}\text { Contribuição da vivência acadêmica em } \\
\text { um centro de convivência de saúde } \\
\text { mental na formação do enfermeiro. }\end{array}$ & $\begin{array}{l}\text { As oficinas de enfermagem servem como estratégias para o desenvolvimento crítico acerca } \\
\text { da RP. Contribuindo de forma significante para um exercício de competência. Essas, } \\
\text { permitem um espaço de ressocialização e descontroem o estereótipo sobre a doença mental. }\end{array}$ \\
\hline $\begin{array}{lr}\text { MAGNAGO } & \text { C } \\
\text { TAVARES } & \text { CMM } \\
\text { (2012). } & \end{array}$ & $\begin{array}{l}\text { O ensino de enfermagem psiquiátrica nas } \\
\text { Universidades Públicas do Estado do Rio } \\
\text { de Janeiro }\end{array}$ & $\begin{array}{l}\text { Os cursos dão condições para o aluno rediscutir a significação da loucura, rompendo com o } \\
\text { estigma de preconceitos e promovendo articulação entre teoria e prática. Todos os } \\
\text { elementos são essenciais para o processo da formação do enfermeiro e para o prestar } \\
\text { cuidado humanizado. }\end{array}$ \\
\hline $\begin{array}{l}\text { TAVARES CMM, et al. } \\
\text { (2014). }\end{array}$ & $\begin{array}{l}\text { Cuidado no hospital psiquiátrico sob a } \\
\text { ótica da equipe de enfermagem }\end{array}$ & $\begin{array}{l}\text { O profissional da enfermagem deve investir no papel de promover o cuidado diante do } \\
\text { contexto da RP. A enfermagem psiquiátrica deve ter como foco o desempenho do cuidado } \\
\text { como prática social de forma individualizada com cada portador de doença mental e } \\
\text { promovendo o convívio social. }\end{array}$ \\
\hline $\begin{array}{l}\text { SINIAK DS, et al. } \\
\text { (2013). }\end{array}$ & $\begin{array}{l}\text { Relato de experiência de estágio de } \\
\text { docência na área de Enfermagem } \\
\text { Psiquiátrica me Saúde Mental. }\end{array}$ & $\begin{array}{l}\text { E importante que as IES retomem sobre a essência do ensino para a RP, de modo a } \\
\text { caracterizar o cuidado psicossocial como novo modelo de atenção em saúde mental, } \\
\text { trabalhando a perspectiva antimanicomial. }\end{array}$ \\
\hline
\end{tabular}

REAS/EJCH | Vol.Sup.n.45 | e2631 | DOI: https://doi.org/10.25248/reas.e2631.2020 Página 5 de 10 


\begin{tabular}{|c|c|c|}
\hline $\begin{array}{l}\text { VARGAS D, et al. } \\
(2014) .\end{array}$ & $\begin{array}{l}\text { Centros de atenção psicossocial } \\
\text { álcool/drogas: inserção e práticas dos } \\
\text { profissionais de enfermagem }\end{array}$ & $\begin{array}{l}\text { A prática e modo de reinserção do profissional de enfermagem no CAPS ad mostram que } \\
\text { esse novo modelo de cuidado passa por uma transição, onde antes exercia funções } \\
\text { controladoras e punitivas. É apontado que a transição ainda esta em processo nesses } \\
\text { serviços e que é importante a educação dos profissionais com as perspectivas da RP. }\end{array}$ \\
\hline $\begin{array}{l}\text { OLIVEIRA LC, et al. } \\
(2015) .\end{array}$ & $\begin{array}{l}\text { Cuidar humanizado: descobrindo as } \\
\text { possibilidades na prática da enfermagem } \\
\text { em saúde mental }\end{array}$ & $\begin{array}{l}\text { Com a RP a enfermagem sai de uma prática de cuidado manicomial para os novos e } \\
\text { desconhecidos princípios da saúde mental. É evidente que a humanização em saúde mental } \\
\text { significa colher, ouvir e proporcionar respostas positivas. }\end{array}$ \\
\hline $\begin{array}{l}\text { GUIMARÃE NA, et al. } \\
(2015) \text {. }\end{array}$ & $\begin{array}{l}\text { Changes in mental health care due to the } \\
\text { psychiatric reform: nursing professional' } \\
\text { perceptions. }\end{array}$ & $\begin{array}{l}\text { Nas últimas décadas tem ocorrido mudanças radicais no atendimento ao paciente mental, } \\
\text { esta mostrou benefícios quanto o fechamento dos hospitais manicomiais, qualificação do } \\
\text { cuidado e profissionais e novas formas de cuidado em saúde mental onde procura romper } \\
\text { com o modelo manicomial para o psicossocial promovendo a reintegração social, autonomia } \\
\text { e cidadania. }\end{array}$ \\
\hline $\begin{array}{ll}\text { SOUZA } & \text { MC } \\
\text { AFONSO LM (2015). }\end{array}$ & $\begin{array}{l}\text { Saberes e práticas de enfermeiros na } \\
\text { saúde mental: desafios diante da } \\
\text { Reforma Psiquiátrica }\end{array}$ & $\begin{array}{l}\text { Os enfermeiros percebem ainda que existem lacunas entre o conhecimento teórico e prático } \\
\text { que é desenvolvido durante a formação e os saberes necessários para atuar nos novos } \\
\text { serviços substitutivos. }\end{array}$ \\
\hline $\begin{array}{l}\text { MAFTUM MA, et al. } \\
(2017) .\end{array}$ & $\begin{array}{l}\text { Mudanças ocorridas na prática } \\
\text { profissional na área da saúde } \\
\text { mental frente à reforma psiquiátrica } \\
\text { brasileira na visão da equipe } \\
\text { de enfermagem }\end{array}$ & $\begin{array}{l}\text { Com essas mudanças ocorridas com a RP no papel da enfermagem em saúde mental, o } \\
\text { mesmo deixa de exercer o papel limitante aos sinais vitais e passa a desempenhar papel } \\
\text { importante no cuidado de forma autônoma, nesses novos serviços de saúde mental exigir } \\
\text { que o profissional tenha uma atitude diferenciada e focada no cuidado humanizado. }\end{array}$ \\
\hline $\begin{array}{l}\text { XAVIER MS, et al. } \\
(2017) .\end{array}$ & $\begin{array}{l}\text { Compreensão de enfermeiras atuantes } \\
\text { em saúde mental sobre a internação } \\
\text { compulsória e involuntária }\end{array}$ & $\begin{array}{l}\text { É visto que há uma dificuldade na comunicação dos serviços preconizados pela RP que } \\
\text { compõem as redes de atenção a saúde mental. É considerado importante trabalhar ações } \\
\text { de educação permanente enfatizando temas como comunicação entre as redes de atenção } \\
\text { em saúde mental de acordo com os preceitos da RP. }\end{array}$ \\
\hline
\end{tabular}

Fonte: Estevam AS, et al., 2018.

REAS/EJCH | Vol.Sup.n.45 | e2631 | DOI: https://doi.org/10.25248/reas.e2631.2020 Página 6 de 10 


\section{DISCUSSÃO}

É possível observar através dos artigos que há uma grande abordagem da importância da formação dos novos enfermeiros generalistas, tendo como base os princípios da RP, ressaltando a importância que os trabalhos na saúde mental possuem para a educação continuada da equipe de enfermagem e dos profissionais que têm contato com essa população.

Nas últimas décadas têm ocorrido mudanças radicais no atendimento ao paciente mental, esta mostrou benefícios como o fechamento dos hospitais manicomiais, qualificação do cuidado e dos profissionais e novas formas de cuidado em saúde mental; no qual procura romper com o modelo manicomial alterando para o psicossocial, promovendo a reintegração social, autonomia e cidadania. É importante que esta discussão sobre a RP e seus novos cuidados seja trabalhada no curso da graduação ampliando o conhecimento do profissional sobre o novo cenário da saúde mental (GUIMARÃES AN, et al., 2015). Contudo, o conhecimento em saúde mental percorre um caminho que vai ao encontro do almejado pela reforma psiquiátrica, principalmente no tocante aos novos serviços e a necessidade do trabalho articulado, com o intuito de minimamente garantir assistência integral e digna aos usuários e a suas respectivas famílias, garantindo respeito a cidadania e autonomia do sujeito.

Entretanto, a localização inadequada das instituições constituintes e a ausência de capacitação adequada dos profissionais de saúde, são fatores preocupantes e dependem de pretensões políticas. Além disso, existe uma falha inegável do sistema de referência e contrarreferência. Pontos que necessitam de empenho para melhor adequação, seguindo os preceitos da RP (MARINHO AM, et al., 2011).

Com essas mudanças do modelo de cuidar em saúde mental, foi visto que os cursos da graduação dão condições para o aluno rediscutir a significação da loucura, rompendo com o estigma de preconceitos e promovendo articulação entre teoria e prática. Todos os elementos foram apontados pelos reformistas como essenciais para o processo da formação do enfermeiro e para que os mesmos sejam capazes de prestar cuidado humanizado e produzindo novos modelos de cuidado na saúde mental (MAGNAGO C e TAVARES $M, 2012$ ).

Os trabalhadores estão cada vez mais conscientes da urgente necessidade de lutar por melhores condições no setor de trabalho e assistência. Além disso, existe a necessidade do fortalecimento do trabalho interdisciplinaridade pautado na reabilitação psicossocial, ainda que muitas instituições e percepções isoladas insista em persistir em práticas baseadas no modelo biomédico. Assim, pressupõe-se que a apego ao modelo flexineriano resiste pelos resquícios de uma formação acadêmica biologicista, em que não havia empenho em executar práticas humanizadas e a assistência à saúde fora do ambiente hospitalar encontrava-se fora de cogitação (MARINHO AM, et al., 2011).

Os enfermeiros acabaram percebendo que existem lacunas entre o conhecimento teórico e prático que é desenvolvido durante a formação e os saberes necessários para atuar nos novos serviços substitutivos. Em alguns momentos ainda pode ser perceptível que algumas práticas ainda estão ligadas as tradicionais, porém é visto que existe uma dificuldade para a concretização das novas políticas na prática mostrando a necessidade de capacitações e atualizações (SOUZA MC; AFONSO MLM, 2015). As oficinas de enfermagem, por exemplo, servem como estratégias para o desenvolvimento crítico acerca da RP. Este contribui de forma significante para um exercício de competência, e a partir dessa oficina acadêmica trabalha-se a desmistificação e redução do preconceito sobre a loucura e também na mudança do modelo manicomial para o de atenção psicossocial. Essas oficinas permitem um espaço de ressocialização e descontroem o estereótipo sobre a doença mental (LOPES CV; REINALDO AMS, 2012).

O profissional da enfermagem deve investir no papel de promover o cuidado diante do contexto da RP. A enfermagem psiquiátrica deve ter como foco o desempenho do cuidado como prática social, respeitando e trabalhando de forma individualizada com cada portador de doença mental e promovendo o convívio social (TAVARES CMM, et al., 2014).

A partir desse momento de transição de cuidado durante a RP, a enfermagem foi posta como protagonista desse cuidado, pois a mesma participou integralmente da consolidação desse modelo de cuidado mostrando também competência científica e teórico/prático para exercer essa função. 
Com essas mudanças ocorridas com a RP no papel da enfermagem em saúde mental, o mesmo deixa de exercer o papel limitante aos sinais vitais, alimentação e higiene e passa a desempenhar papel importante e ativo no cuidado de forma autônoma, nesses novos serviços de saúde mental se torna necessário exigir que o profissional tenha uma atitude diferenciada e focada no cuidado humanizado (MAFTUM MA, et al., 2017).

A prática e modo de reinserção do profissional de enfermagem mostram que esse novo modelo de cuidado passa por uma transição, onde antes exercia funções controladoras e punitivas, porém ao assumirem papel protagonista na RP brasileira exigiu-se que houve essa transição. Então nesse novo modelo é pressuposto a quebra do velho modelo para uma nova forma de cuida. É apontado que a transição ainda está em processo nesses serviços e que é importante a educação dos profissionais com as perspectivas da RP (VARGAS D, et al., 2014).

Com a RP a enfermagem sai de uma prática de cuidado manicomial para os novos e desconhecidos princípios da saúde mental. É evidente que a humanização em saúde mental significa acolher, ouvir e proporcionar respostas positivas. Os enfermeiros reconhecem que existem vários desafios para serem enfrentados no cuidar humanizado e que tais desafios decorrem dos problemas na sua prática, como fragilidade e habilidade até a falta de recursos humanos ou outros e demandas das atividades (OLIVEIRA LC, et al., 2015).

Azevedo DM e Santos AT (2012) mostram que os enfermeiros que foram entrevistados em sua pesquisa alegam conhecer a RP e a importância desta no cuidado em saúde mental na ESF. As divergências e discordâncias encontradas acabam sugerindo fragilidade entre a articulação CAPS e ESF, interferindo na assistência e demonstrando desconhecimento sobre o matriciamento.

Além disso, também é visto que há uma dificuldade na comunicação dos serviços preconizados pela RP que compõem as redes de atenção a saúde mental. E com essa nova forma de cuidar a enfermagem acaba pensando, também em discussões que promovam qualidade de assistência nas internações compulsórias e involuntárias. É considerado importante trabalhar ações de educação permanente enfatizando temas como comunicação entre as redes de atenção em saúde mental de acordo com os preceitos da RP (XAVIER MS, et al., 2017).

Cecilio LCO e Reis AAC (2018), tratam da falta de comunicação da rede básica como um dos desafios atuais da Atenção Básica à Saúde (AB). Enquanto pensamos no modelo de Centos de Atenção Psicossocial como uma das propostas da RP, estando eles inseridos na $A B$, como forma de prevenção e reabilitação do usuário, podemos concluir que, a falha de comunicação alcança esse subsistema comprometendo a regulação e atendimento do usuário na sua integralidade.

Os Centros de Atenção Psicossocial (CAPS) possuem um novo caráter de assistência, são unidades de atendimento em saúde mental que oferecem aos usuários um programa de cuidados intensos e individualizados, elaborados por uma equipe multidisciplinar o qual possui um grande significado na inserção do usuário na sociedade. As famílias também acompanham o usuário nos serviços afim de prepará-las para enfrentar a vida social, visto que o núcleo familiar é considerado parte integrante e fundamental no processo de reabilitação. (BRANCO FMFC, et al., 2019).

Apesar do conhecimento do papel da família no processo de reinserção social do paciente de saúde mental, nenhum dos artigos revisados citou esse ponto. Branco FMFC, et al. (2019) aponta a enfermagem como a profissão capaz de realizar um papel fundamental a partir de uma visão holística e integral, através de um amplo conhecimento com fatores precipitados, predisponentes e perpetuantes, tende a realizar cuidados que abrangem os aspectos éticos, culturais, sociais, psicoterápicos, voltado ao paciente, a família e a comunidade.

Ele aponta ainda a Sistematização da Assistência de Enfermagem (SAE) como ferramenta e modelo que permite o enfermeiro buscar uma melhor assistência ao usuário que necessita de cuidados e atenção voltados à Saúde Mental o que permitirá que o mesmo volte ao convívio social (BRANCO FMFC, et al., 2019).Reafirmando assim a importância que a Instituição de Ensino Superior (IES) retome e trabalhem sobre a essência do ensino para a RP, de tal forma que possa caracterizar o cuidado psicossocial como novo modelo 
de atenção em saúde mental, trabalhando a perspectiva antimanicomial preconizadas pela RP (SINIAK DS, et al., 2013). Contudo o ensino não deve se conter somente a graduação, a mesma deve ser procurada através de outras modalidades, seja ela dentro da educação continuada ou permanente.

Destarte, o que outrora foi denominado de cultura manicomial, pode-se apresentar em diferentes configurações, destacado o distanciamento da dimensão subjetiva e existencial do sofrimento mental, em prol de constituição reducionista do sujeito pautada no físico. Portanto, é indispensável que se os profissionais estejam atentos para evitar a replicação estéril de um discurso acerca da prática de saúde mental, sem que de fato seja feita uma reflexão adequada quanto as práticas estabelecidas para o cuidado. Desse modo, a qualidade da assitência prestados tem influência dos conceitos sociais que estão presente no processo de produção do cuidado. O que nos leva a refletir sobre a necessidade de apostar no saber das equipes de saúde mental, pensado em uma perspectiva humanista, criativa, reflexiva e imaginativa, que leva como tendência um cuidado refletido em processos dinâmicos e em constante modificação (MUNIZ MP et al., 2015).

\section{CONSIDERAÇÕES FINAIS}

Esta pesquisa conclui que com essa mudança de panorama do cenário da saúde mental a enfermagem assumiu papel importante tanto na consolidação da RP quanto nas mudanças das práticas em saúde mental, fazendo assim da enfermagem uma profissão tida atualmente como protagonista em todos os serviços substitutivos de atenção psicossocial. Mas, para que isso continue ocorrendo e a enfermagem continue exercendo essa posição, necessita que ela ensine desde a graduação sobre os preceitos da RP e haja incentivo da educação continuada dos profissionais, pois foi visto em alguns estudos que apesar dos profissionais saberem sobre a RP, na prática eles acabam realizando cuidados que são pautados no modelo manicomial. Salienta-se a necessidade de pesquisas que discorram sobre as dificuldades do cuidado humanizado aos pacientes de saúde mental.

\section{REFERÊNCIAS}

1. AZEVEDO DM, SANTOS AT. Ações de saúde mental na atenção básica: conhecimento de enfermeiros sobre a reforma psiquiátrica. Revista de Pesquisa: Cuidado é Fundamental (online), 2012, 4(4), 3006-3014.

2. ARGILES CT, et al. Redes de sociabilidade: construção a partir do serviço de residência terapêutico. Ciência e Saúde Coletiva, 2013, 18(7), 2049-2058.

3. BRASIL, Ministério da Saúde. Secretaria-Executiva. Secretaria de Atenção à Saúde. Legislação em saúde mental: 1990-2004. Brasília: Ministério da Saúde, 5. ed., 2004.

4. BRANCO FMFC, SILVA JBS, DUTOK CMS, NETO, TCBN. Percepção dos familiares de pessoas com esquizofrenia acerca da doença. Revista Eletrônica Acervo Saúde / Electronic Journal Collection Health, 2019, 11(12) e944.

5. CECILIO LCO, REIS AAC. Apontamentos sobre os desafios (ainda) atuais da atenção básica à saúde. Cadernos de Saúde Pública [online]. 2018, v. 34, n. 8 [Accessed 13 February 2020], e00056917.

6. ESPERIDIÃO E, et al. A enfermagem psiquiátrica, a ABEn e o departamento cientifico de enfermagem psiquiátrica e saúde mental: avanços e desafios. Revista brasileira de enfermagem. Brasília, 2013, 66(17), 1-6.

7. HIRDES A A. Reforma Psiquiátrica no Brasil: Uma (re)visão. Revista Ciência e Saúde Coletiva. Rio de Janeiro, 2009, 14(1), 297-305.

8. LOPES CV, REINALDO AMS. Contribuição da vivencia acadêmica em um centro de convivência de Saúde Mental na formação do Enfermeiro. Cogitare Enfermagem, 2012, 17(4), 775-778.

9. LIMA DWC, et al. A escuta terapêutica no cuidado clínico de enfermagem em Saúde mental. Texto Contexto Enfermagem, 2015, 24(1), 154-160.

10. GUIMARÃES AN, et al. Changes in Mental Health care due to the Psychiatric Reform: Nursing professionals' perceptions. Ciência Cuidado e Saúde, 2015, 14(1), 830-838.

11. MENDES KS, et al. Revisão integrativa: método de pesquisa para a incorporação de evidências na saúde e na enfermagem. Texto contexto em Enfermagem, 2008,17(4), 758-764.

12. MAGNAGO C, TAVARES CMM. O ensino de Enfermagem psiquiátrica nas Universidades Públicas do Estado do Rio de Janeiro. Revista Eletrônica de Enfermagem (online), 2012, 14(1), 50-58.

13. MAFTUM MA, et al. Mudanças ocorridas na prática professional na área da saúde mental frente à reforma psiquiátrica brasileira na visão da equipe de enfermagem. Revista de Pesquisa: Cuidado é Fundamental (online), 2017, 9(2), 309314.

14. MARINHO AM, et al. Reflexões acerca da reforma psiquiátrica e a (re)construção de políticas públicas. Revista Mineira de Enfermagem; 15(1): 141-147, jan./mar., 2011. 
15. MUNIZ MP, et al. A assistência de enfermagem em tempos de reforma psiquiátrica. Revista Portuguesa de Enfermagem de Saúde Mental [Internet]. 2015 Jun [citado 2020 Fev 13] ; ( 13 ): 61-65.

16. OLIVEIRA LC, et al. Cuidar humanizado: descobrindo as possibilidades na pratica da enfermagem em saúde em mental. Revista de Pesquisa: Cuidado é Fundamental (online), 2015, 7(1), 1774-1782, 2015.

17. SINIAK DS, et al. Relato de experiência de estágio de docência na área de Enfermagem Psiquiátrica me Saúde Mental. Ciência, Cuidado e Saúde, 2013, 13(3), 593-598.

18. SOUZA MC, AFONSO MLM. Saberes e práticas de Enfermeiros na Saúde Mental: desafios diante da Reforma Psiquiátrica. Gerais: Revista Interinstitucional de Psicologia, 2015,8(2), 332-347.

19. TAVARES CMM, et al. Cuidado no hospital sob a ótica da equipe de enfermagem. Revista Rene, 2014,15(2), 282290.

20. VARGAS D, et al. Centros de atenção pasicossocial álcool/drogas: inserção e práticas dos profissionais de enfermagem. Escola Anna Nery, 2014, 18(1),101-106.

21. XAVIER MS, et al. Compreensão de enfermeiras atuantes em sáude mental sobre a internação compulsória e involuntária. Escola Anna Nery, 2017, 21(3), 1-8. 